\title{
Polyolefin from Commodity to Specialty
}

\section{Mike Chung TC*}

Department of Materials Science and Engineering, Pennsylvania State University, University Park, PA 16802, USA

\section{Introduction}

Polyolefins-including polyethylene (PE), polypropylene (PP), poly(1-butene), poly(4-methyl-1-pentene), ethylene-propylene elastomer (EPR), and ethylene-propylene-diene rubber (EPDM) - are the most widely used commercial polymers, with over 120 million metric tons global annual consumption, or close to $60 \%$ of the total polymer produced in year 2014. The popularity is due to their excellent combination of chemical and physical properties along with low cost, superior processability, and good recyclability. By controlling crystallinity and molecular weight, polyolefins with a wide range of thermal and mechanical properties have been produced for wide range of commercial applications. The products are presented in various forms, such as films, sheets, fibers, and even wax and viscous liquid. They are greatly influencing our day-to-day life, and range from common items like bread and garbage bags, milk jugs, bottles, containers, hoses, outdoor-indoor carpets, tires, bumpers and trims to bullet-proof jackets. It is very interesting to note that the simplest molecular structure of linear polyethylene (HDPE) with ultra-high molecular weight (such as the commercial spectrum fiber used in bullet-proof jackets) has near 100\% crystallinity and melting temperature $(\mathrm{Tm}) \sim 140^{\circ} \mathrm{C}$. Its mechanical strength is greater than any known existing materials, including steel, Kelvar, carbon fiber, etc. On the other hand, the ethylene-propylene copolymers, containing about 40-45 mol\% of propylene, are completely amorphous elastomers with the glass transition temperature $(\mathrm{Tg})$ as low as $-65^{\circ} \mathrm{C}$. With its great commercial success, it is very interesting to look back at the evolution of this technology and to refresh the most important discoveries and breakthroughs during the past five decades. It is even more important to think of possible future developments. What are the shortfalls of polyolefin materials that prevent their even wider usage in many areas currently occupied by other polymers that are much more expensive and less environmentally friendly materials? What are the needed technologies? How much has been done to address the technological challenges?

Polyolefin development dates back to the early 1930s with the production of low-density polyethylene (LDPE) by free radical initiators. The polymerization required very high temperature and pressure conditions, and the polymer contains long and short branches that affect the polymer chain packing. Thus, LDPE exhibits relatively low density, low crystallinity, low melting point, and low mechanical strength. In the early 1950s, the historic discovery of Professor Karl Ziegler at the Max Planck Institute in Germany by using early transition metal catalyst opened up the new age in the preparation of polyolefin, as well as the new frontier for the utility of transition metal coordination chemistry in the catalytic chemical reactions. The ethylene polymerization mediated by $\mathrm{TiCl}_{4}$ and AlEt 3 can take place at ambient temperature and low-pressure conditions to produce linear polyethylene (HDPE) with high density, high crystallinity and high melting point. Professor Giulio Natta realized that the Ziegler catalyst is capable of stereoregularly polymerizing $\alpha$-olefins into the polymer with stereoregular repeating units. The catalyst controlled isotactic structure is responsible for the excellent thermal and mechanical properties of isotactic polypropylene (i-PP). Ziegler and Natta were jointly awarded the Nobel Prize for Chemistry in 1963 for their roles in the start of the "Golden Age" of polymer science. The tremendous research efforts were generating the discovery of catalysts with superior activity and stereospecificity, as well as leading to economically viable production processes and product developments. In the late 1970s, Kaminsky and Sinn discovered a new class of homogeneous (single-site) Ziegler-Natta catalyst, based on metallocene/methylaluminoxane (MAO), which offers tremendous advantages in understanding the polymerization mechanism and allows the design of catalyst to prepare new polymers (especially copolymers) with narrow molecular weight and composition distributions. Polyolefin technology and industry have been changing the landscape by broadening the monomer pool and introducing new high performance products and new applications. This single-site catalyst technology was further expanded to late transition metals. In 1990s, Brookhart et al. reported the success of achieving high polymers with exceptionally high catalyst activity by some well-designed $\mathrm{Ni}, \mathrm{Pd}$, $\mathrm{Co}, \mathrm{Fe}$ catalysts, containing bulky diimine ligand (non-metallocene type). A highly branched polymer structure was formed with the degree of branching controlled by reaction conditions. Because of the less oxophilic nature of late transition metal, the catalyst system is also more tolerant to functional groups. This catalyst, astonishing us with its power and scope of transition metal coordination polymerization, truly elevates polyolefin catalysis to another level and opens the door for new products and applications.

Overall, the past more than a half-century of development of polyolefin technology has been centered on the discovery of new catalyst systems. The successful catalyst systems were also accompanied with new production processes, such as gas phase, suspension, solution, etc., for industrial-scale production. Despite the great scientific and commercial success, there are shortfalls of polyolefin materials that prevent their even wider usage in many areas currently occupied by other polymers that are much more expensive and less environmentally friendly materials. Polyolefin material is known to be inadequate in coatings, blends, and composites, where adhesion, compatibility, wettability, or reactivity are paramount. Due to lack of polar functional group, polyolefin exhibits poor interaction with other materials, such as pigments, paints, clay, glass fibers, metals, carbon black, and most polymers. Most of these difficulties should be resolved by introducing polar functionality or by grafting suitable polar monomers to polyolefin, which could enhance interactions between polyolefin and other materials. In fact, since the commercialization of HDPE and PP in the 1950s the functionalization of polyolefin has been an area of intense interest in both academic and industrial communities. The perpetual interest is driven by an impetus to improve

${ }^{*}$ Corresponding author: Mike Chung TC, Professor, Department of Materials Science and Engineering, Pennsylvania State University, University Park, PA 16802, USA, Tel: 814-863-1394; E-mail: chung@ems.psu.edu

Received April 02, 2015; Accepted April 06, 2015; Published April 12, 2015

Citation: Mike Chung TC (2015) Polyolefin from Commodity to Specialty. J Material Sci Eng 4: e111. doi:10.4172/2169-0022.1000e111

Copyright: (c) 2015 Mike Chung TC. This is an open-access article distributed under the terms of the Creative Commons Attribution License, which permits unrestricted use, distribution, and reproduction in any medium, provided the original author and source are credited. 
on the poor interactive properties of polyolefins and apply them to higher value products. Despite significant research efforts, both direct and post-polymerization approaches have yielded limited success, leading only a few commercially-available technologies and products. The direct process could be an ideal one (involving an only one-step reaction) if the copolymerization reaction with functional monomers would be as effective and straightforward as the corresponding homopolymerization reaction. Unfortunately, some fundamental issues of catalyst poisoned by functional (polar) groups have prevented serious consideration of the direct process for commercial application. So far, most of the commercial functionalization processes are based on post-polymerization. Chemical modification of the pre-formed polyolefin homopolymers has been usually carried out in situ during the fabrication process to reduce the production cost, as well as to relieve the significant concern (in many cases) of reducing processibility of polyolefin after functionalization reaction. However, the combination of the inert nature of polyolefin (requiring highly energetic reaction conditions) and a very short reaction time (during the processing) causes a great difficulties in controlling polymer composition and structure.

In the past more than two decades, the combination of advance catalyst technology and new functionalization methods has brought new light to this difficult area with many promising results. My interest in functional polyolefin dated back to 1984, when I was just starting my early industrial career at Exxon, Corporate Research, maybe I was in the right place and at the right time to benefit from some early exciting development of metallocene catalysis by my colleagues in Exxon Chemical Co. The potential of metallocene catalyst in the copolymerization reactions, exhibiting superior comonomer incorporation and well-controlled copolymer structures, intrigued me with the initial thought of searching a facile "reactive" comonomer that could be effectively incorporated into polyolefin. Then, the incorporated "reactive" groups can be selectively interconverted to polar functional groups, or preferably be transformed to initiators for graft-from polymerization of functional monomers. In other words, the basic idea is to circumvent the chemical difficulties in both direct and post-polymerization processes by designing a reactive copolymer "intermediate" that can be effectively synthesized and subsequently interconverted to functional polymer, as well as leading to the preparation of a polyolefin graft polymer having a polyolefin backbone and several functional (polar) polymer side chains. After more than two decade-long efforts of my postdoctors and graduate students in my research group at Penn State University pursuing this idea of the "reactive" comonomer approach, which is coincidental with the continuous evolution of metallocene catalysis, we have developed three useful reactive comonomer systems, i.e. organoborane, p-methylstyrene, and divinylbenzene. In addition, the chemistry has been applied to "reactive" chain transfer-agents to incorporate a reactive group at the polymer chain end and consequently to prepare polyolefin functional diblock copolymers. The well-defined polymerization mechanism of metallocene catalysis leads to a precise control of chain transfer reaction. With the design of a chain transfer agent containing a reactive group, the in situ chain transfer reaction produces the polymer having a terminal reactive group. In turn, this reactive end group opens up a lot of possibility to produce new polyolefin products, including diblock copolymers and long chain branching polymers. They present potentials for polyolefins in high-value, specialty applications. Some functional PP polymers with polar (or ionic) groups have shown high dielectric constants and breakdown strengths, they are useful in high energy density PP film capacitors. PE graft copolymer containing several sulfonated poly(arylene ethersulfone) (s-PAES) side chains shows a unique combination of hydrophobicity and semi-crystallinity and also offers high proton conductivity and stable (non-swellable) matrix. They are cost-effective proton exchange membranes for fuel cell applications. Recently, we have also developed a new polyolefinbased oil super-absorbent polymer (oil-SAP) that exhibits high oil absorption capability (up to 45 times of its weight), fast kinetics, easy recovery from water surface, and no water absorption. The recovered oil-SAP solid is suitable for regular refining process (no pollutants and no wastes). This new polyolefin based oil-SAP technology shall dramatically reduce the environmental impacts from oil spills and recover most of precious natural resource. 